\title{
Systems of Pain/Networks of Resilience (Nebraska)
}

\author{
Meghan Moe Beitiks
}

VIDEO LINK: https://vimeo.com/241505499

Systems of Pain/Networks of Resilience (Nebraska) is a performance for a camera. Chapters of the work have developed in Omaha, Nebraska, upstate New York, Galesburg, Illinois and Santa Fe, New Mexico. (Nebraska) is the first chapter, originally performed live at the Bemis Center for Contemporary Arts. Within the project, pain is understood as trauma, loss, disruption-everything from polluted landscapes to neglect, from abusive relationships to work stress. Resilience is similarly embraced in an expansive, cross-disciplinary way, invoking everything from the ability of an ecosystem to adapt to disruption, to a human's ability to cope with traumatic events.

(Nebraska) depicts the actions of an androgynous person in an environment of disrupted domestic objects. Performed by artist Meghan Moe Beitiks, this person labors, moves things, fixes things, sits up straight, slouches, interacts with the environment around them. The "stuff" in the space includes items one might expect to find within a still life painting: a tablecloth, tangerines, a vase, knives. Within the work, this seemingly familiar domestic composition from both homes and art histories is perpetually disrupted. As viewers, our experience of the video is both guided and interrupted by the audio from various interviews, and the narration of a professional Audio Describer.

Audio description is frequently used to make film and video accessible for the vision impaired or blind. We hear literal descriptions of actions as the piece progresses, sometimes as the actions occur, sometimes well after the fact. In common practice, many audio descriptions are read quickly, in order to fit between lines of dialogue or other audio. Here they are incorporated more deliberately into the pacing of the work. For the sighted, the experience of hearing a description of what they are seeing while they are seeing it may disrupt the default process of perception-might bring them more fully into the present, into awareness of their own process of observation.

This experience of observation proves important to the work. Beyond the literal audio descriptions, we hear audio clips from interviews. In one prolonged interview clip, a woman describes the experience of flashbacks as a symptom of PTSD, and the work her friends do to bring her out of them, while Beitiks arranges and rearranges items on a table. For the interviewee, recovery is a process of observation and description, of reminding her where she is, and what her relationship is to the place and things around her. It's important that her friends do not mock, stigmatize or label her for her symptoms, but rather see themselves as agents in her recovery. This healing process is unique to the speaker, but central to the questions within Systems of Pain/Networks of Resilience. 
Without assuming a normative or universal healing process for our many and varied pains and traumas, how can interrelatedness assist in our recovery processes? Could the simple act of observation-of being present with the nonhuman-be a useful tool in healing from traumatic and toxic experiences? Can a commitment to observation help humans transcend their tendencies toward prejudice and stigma-themselves traumatizing agents? These are the questions the project asks.

In developing every chapter of the work, Beitiks interviewed individuals with personal and professional processes of recovery, be they emotional or physical, grounded in animal behavior or ecological restoration. The quotes we ultimately hear from these interviews are deliberately ambiguous in nature-it's often unclear whether the speaker is a sociologist, ecologist, or survivor of abuse. This ambiguity means that we can "hear" similarities and connections across disciplines, processes, species. It also means that the quotes, regardless of origin, become associated with the performance we're seeing onscreen-the actions of the person in the disrupted landscape. The narration of the work swaps back and forth between the literal description of the action, and the narratives of trauma and recovery in the interviewees' voices.

A final quote from an interviewee describes vulnerability as a term that is both emotional and scientific-used not only to describe the parts of us that are most susceptible to pain, but the communities that are most likely to be impacted by climate change-the species, ecosystems, individuals and societies that are most likely "to be hurt." Observational awareness requires that we consider the limits of our own perceptions-consider whose needs we might be overlooking, regardless of training, material or origin.

For (Nebraska), the Audio Descriptions were created by Emily Smith Beitiks. The original performance was created with technical support from Tara Kaufman, and filmed by MakeBelieve Studios. 